\title{
Rapid reversibility of Takotsubo cardiomyopathy
}

\author{
Fathia Mghaieth Zghal, Ramy Trabelsi, Sihem Mbarki, Mohamed Sami Mourali, Rachid Mechmeche
}

Rabta University Hospital, Faculty of Medicine, University of Tunis El Manar, Tunis, Tunisia

Email: fathiazghal@yahoo.com

Received 22 September 2011; revised 7 November 2011; accepted 24 November 2011

\begin{abstract}
Background: In Takotsubo cardiomyopathy (TCM), left ventricular dysfunction is usually reversible within a few weeks. Complete recovery can however be very rapid. We report the case of Mrs. TA, a 53-yearold patient who had a history of hypertension treated with atenolol and captopril. She was admitted with the diagnosis of non ST segment elevation myo-cardial infarction. The electrocardiogram showed anterior sub-and the troponinI level was $3.48 \mathrm{mg} / \mathrm{l}$. Echocardiography revealed a left ventricular (LV) ballooning and LV ejection fraction was $36 \%$. A recent emotional stress was mentioned. TCM was suspected and emergency angiography showed apical ballooning with a normal coronary angiogram. There was a complete recovery in $\mathbf{7 2}$ hours with a normalization of regional and global LV function at echocar-diography and angiography. Conclusion: There is a large variability in TCM evolution aspects probably due to a variable physiopathological mechanism which remains to be clarified.
\end{abstract}

Keywords: Apical Ballooning; Takotsubo Cardiomyopathy; Early Recovery

\section{INTRODUCTION}

Takotsubo cardiomyopathy (TCM) is a clinical entity characterized by an acute left ventricular (LV) systolic dysfunction triggered by an emotional or physical stress factor. It is usually reversible within a few weeks to months. We describe a case of TCM with initial severe impairment of left ventricular function that was totally regressive within 72 hours.

\section{CASE REPORT}

Mrs. TA, a 53 year old patient, postmenopausal, having only a systemic hypertension as a cardiovascular risk factor which had been treated by atenolol and captopril, was admitted for a suspected acute coronary syndrome due to an inaugural pseudo-anginal chest pain. A recent emotional stress in a context of family conflict was men- tioned but there was no recent flu like symptoms. On examination, blood pressure (BP) was 100/60 $\mathrm{mmHg}$, the heart rate was $60 \mathrm{bpm}$, cardiac auscultation was normal and there were no signs of heart failure. The electrocardiogram showed a negative $\mathrm{T}$ wave in V1-V6, DI, DII and Avl (Figure 1). The peak of troponin I level was $3.48 \mathrm{mg} / \mathrm{l}$. Echocardiography revealed an LV apical ballooning with an akinesia of the apical two thirds of $\mathrm{LV}$ walls circumferentially and LV ejection fraction (LVEF) was $36 \%$ by biplane Simpson method. Angiography, without any medical provocative test, performed at the 12th hour found a normal coronary angiogram (Figure 2) and LV apical ballooning on ventriculography (Figure 3). The diagnosis was then rectified and TCM was decided as the final diagnosis. The pain was relieved in a few hours. The patient did not experience any dyspnea or rhythm disturbance. The next day, the echocardiography showed a decisive improvement of regional and global LV function. Control at 72 hours objectified a normalization of ECG and troponin level as well as a complete recovery of LV function (LVEF: 65\%) assessed by ultrasounds and angiography (Figure 4). On follow-up to one year with the same medical treatment (atenolol and captopril) there was no recurrence of TCM or other cardiovascular event.

\section{DISCUSSION}

Takotsubo cardiomyopathy (TCM) is a transient left ventricular dysfunction secondary to an emotional or physical stress and characterized by a left ventricular (LV) apical ballooning with wall motion disorders with a distribution that does not follow a specific coronary artery anatomy [1].

Although it can manifest by a cardiogenic shock or a ventricular fibrillation [1,2], in most cases (as in our patient), its clinical presentation mimics an acute coronary syndrome (ACS). However, some clinical, echocardiographic, angiographic, and especially evolution particularities differentiate TCM from ACS. The evolution of TCM reported in various studies, is characterized by a regression of wall motion abnormalities and gradual restoration of LV function until complete recovery is achieved [2]. 

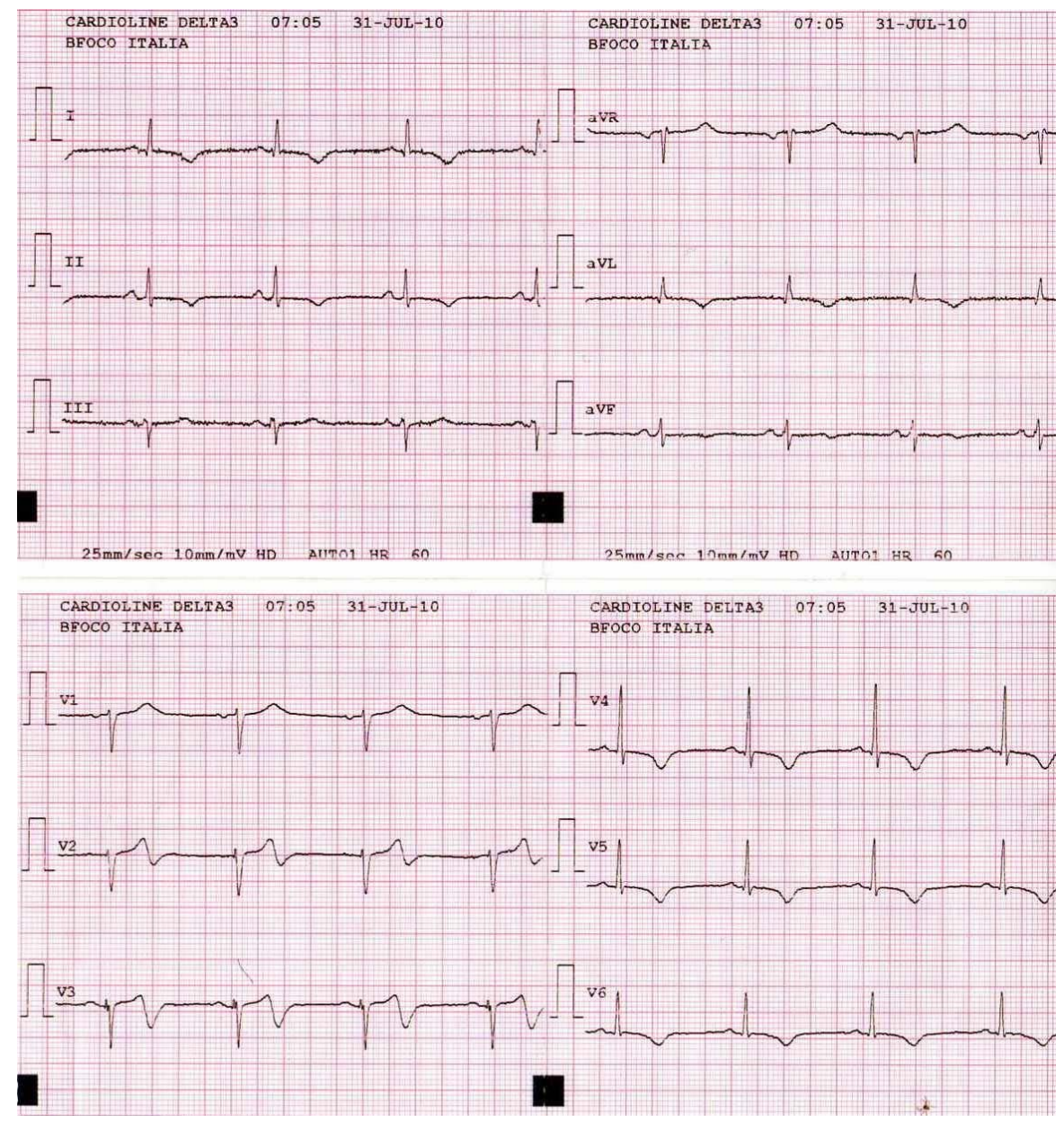

Figure 1. Twelve leads electrocardiogram on admission: sub-epicardial ischemia in anterior leads.

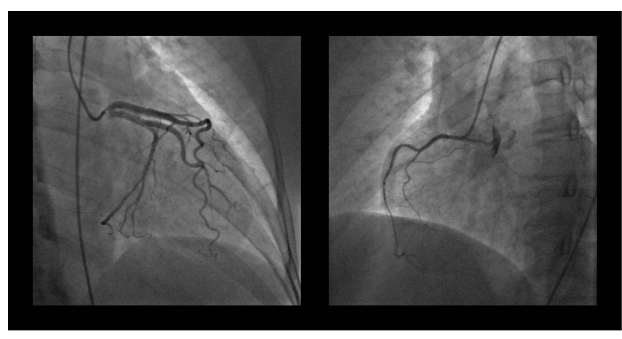

Figure 2. Normal coronary angiogram on adssion. At left: left coronary artery, at right: rit coronary artery.

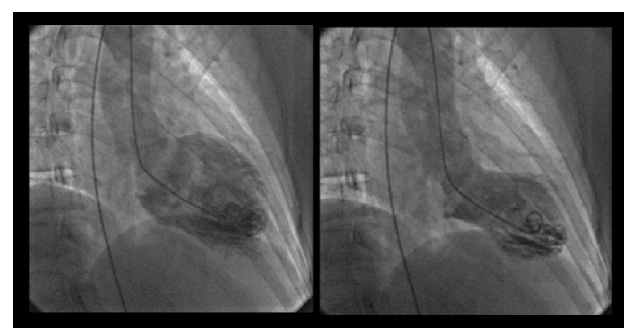

Figure 3. Ventricular angiography on admission. At left: diastole, at right: systole; apical ballooing.

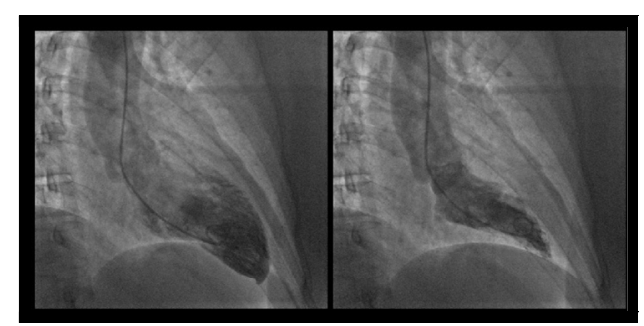

Figure 4. Ventricular angiography at day 3. At left: diastole, at right: systole; normal ventricular systolic function.

Complications may occur in approximately $20 \%$ of cases. They include cardiogenic shock, heart failure, rhythm disorders, the formation of a thrombus in the LV, free wall rupture and death. In-hospital mortality reported in the studies of Gianni et al. [2], and Donohue et al. [3], was $1 \%$ and $3.2 \%$. The long-term prognosis of TCM is much better than that of ACS. Recurrence can be observed in $2 \%-3 \%[2]$. The reversibility of acquired disorders is usually rapid. Regression of electrocardiographic signs and normalization of cardiac biomarkers can be seen in 
the first three days $[4,5]$. The normalization of myocardial contractile function is usually achieved after 1 to 4 weeks [6]. In the study of Wittstein et al. [4], the median LVEF on admission, after 3 days - 7 days and 2 weeks 4 weeks was respectively $20 \%, 45 \%$ and $60 \%$. A slower and delayed evolution beyond one year was however reported. Kurisu et al. [7] reported two cases of elderly female patients, with a TCM after pace-maker implantation, who had persistent LV dysfunction after 2 to 4 months of follow-up. These authors [8] in contrast, described a case of emotional related TCM in a context of family conflict with typical features on ventriculography which were reversible in 41 hours. The evolution in our patient was also very rapid and there was a complete regression of the $\mathrm{LV}$ wall motion impairment of and the LVEF returned to a normal value on echocardiography and angiography within 72 hours. In the study on the City of Minneapolis in 2005, including 22 patients with TCM [9], the delay of recovery of LV function documented by magnetic resonance imaging and echocardiography was $6 \pm 3$ days; shorter than classically described. Probably the most rapid favorable outcome was described by Eitel et al. [10], in a 76 year old woman with a full recovery of contractile function assessed by magnetic resonance imaging in 36 hours. These different TCM evolution patterns may be related to different pathophysiological mechanisms contribution (catecholamine induced myocardial stunning, transient epicardial vasospasm, coronary microvascular dysfunction and transient LV obstructions are discussed). The role of medical management can also be decisive. Our patient was taking a beta-blocker and an angiotensin-converting enzyme inhibitor prior to the occurrence of TCM for hypertension; these two therapies are known to have a favorable action on myocardial function and beta-blockers which counteract the effect of catecholamine seem to be a main treatment in TCM. The patient described by Eitel et al. [10] received alpha-1-adrenergic blocker (urapidil) and nitroglycerine intravenously in addition to a beta-blocker (bisoprolol) and an angiotensin-converting-enzyme-inhibitor (ramipril) which were administered in their highest recommended doses because of an excessive elevated blood pressure. The initial degree of LV function impairment does not seem to be decisive in the subsequent rapidity of evolution, as our patient had a marked LV dysfunction. The role of patient's age also seems secondary since the patients described by Eitel et al. [10] and Kurisu et al. [8] had an advanced age in contrast to our patient.

A better understanding of the causes and pathophysi- ological mechanisms of TCM could better define its clinical, diagnostic, therapeutic characteristics and evolution features.

\section{ACKNOWLEDGEMENTS}

The authors are grateful to Richard Medeiros, Rouen University Hospital Medical Editor, for editing the manuscript.

\section{REFERENCES}

[1] Prasad, A., Lerman, A. and Rihal, C.S. (2008) Apical ballooning syndrome (Tako-Tsubo or stress cardiomiopathy): A mimic of acute myocardial infarction. American Heart Journal, 155, 408-417. doi:10.1016/j.ahj.2007.11.008

[2] Gianni, M., Dentali, F., Grandi, A.M., et al. (2006) Apical ballooning syndrome or Takotsubo cardiomyopathy: A systematic review. European Heart Journal, 27, 15231529. doi:10.1093/eurheartj/ehl032

[3] Donohue, D. and Movahed, M.R. (2005) Clinical characteristics, demographics, and prognosis of transient left ventricular apical ballooning syndrome. Heart Failure Reviews, 10, 311-316. doi:10.1007/s10741-005-8555-8

[4] Wittstein, I.S., Thiemann, D.R., Lima, J.A., et al. (2005) Neurohumoral features of myocardial stunning due to sudden emotional stress. New England Journal of Medicine, 352, 539-548. doi:10.1056/NEJMoa043046

[5] Park, J.H., Kang, S.J., Song, J.K., et al. (2005) Left ventricular apical ballooning due to severe physical stress in patients admitted to the medical ICU. Chest, 128, 296302. doi:10.1378/chest.128.1.296

[6] Desmet, W.J., Adriaenssens, B.F. and Dens, J.A. (2003) Apical ballooning of the left ventricle: first series in white patients. Heart, 89, 1027-1031. doi:10.1136/heart.89.9.1027

[7] Kurisu, S., Inoue, I., Kawagoe, T., et al. (2006) Persistent left ventricular dysfunction in Takotsubo cardiomyopathy after pacemaker implantation. Circulation Journal, 70, 641644. doi:10.1253/circj.70.641

[8] Kurisu, S., Innoue, I., Kawagoe, T., et al. (2007) Documentation of early improvement of left ventricular function in Takotsubo cardiomyopathy. International Journal of Cardiology, 114, e70-e72. doi:10.1016/j.ijcard.2006.07.203

[9] Sharkey, S.W., Lesser, J.R., Zenovich, A.G., et al. (2005) Acute and reversible cardiomyopathy provoked by stress in women from the United States. Circulation, 111, 472479. doi:10.1161/01.CIR.0000153801.51470.EB

[10] Eitel, I., Lücke, C., Behrendt, F., et al. (2010) Full recovery of Takotsubo cardiomyopathy (apical ballooning) in two days. International Journal of Cardiology, 143, e51e53. doi:10.1016/j.ijcard.2008.12.044 